\title{
AGB star models. Results from 1D stellar evolution and multi-dimensional hydrodynamics simulations
}

\author{
Falk Herwig $\dagger$ \\ ${ }^{1}$ Keele Astrophysics Group, School of Physical and Geographical Sciences, Keele University, \\ Staffordshire ST5 5BG, UK \\ email: fherwig@astro.keele.ac.uk
}

\begin{abstract}
In this review I am discussing the current state of simulating the internal evolution of AGB stars. Recent work on AGB stars include the effect of rotation, magnetic fields and internal gravity waves, as well as thermohaline mixing induced by the ${ }^{3} \mathrm{He}+{ }^{3} \mathrm{He}$ pp-chain reaction. Hydrodynamic simulations of the interior convection of AGB stars are now becoming available, giving insights to convective boundary mixing, for example for He-shell flash convection. At very low metallicity convective-reactive events are encountered in AGB stars (as well as in massive stars), and the necessity of hydrodynamic simulations to address this difficult phase of stellar evolution is emphasized.
\end{abstract}

Keywords. Stars: AGB and post-AGB, stars: rotation, stars: magnetic fields

\section{Introduction}

Asymptotic Giant Branch (AGB) stars are the premier nuclear production phase of low- and intermediate mass stars. Their evolution has been extensively studied through one-dimensional, spherically symmetric stellar evolution simulations and numerous reviews are available that summarize the results, including, for example, Iben \& Renzini (1983), Lattanzio (1989), Blöcker et al. (2000) and more recently Herwig (2005).

An important goal of AGB stellar evolution simulations is to provide the thermodynamic evolution that allows the detailed determination of nucleosynthesis and resulting yields, which is for example reviewed by Lattanzio \& Boothroyd (1997). A detailed account of the s process in AGB stars has been provided by Busso et al. (1999). Yields based on stellar evolution tracks have been published, for example, by Forestini \& Charbonnel (1997) and more recently by the Monash group, culminating in a complete yield set for a wide range of initial masses and metallicities including the light elements up to sulfur (Karakas \& Lattanzio 2007). Their results and comparison to previous work reaffirms the two dominant factors in providing accurate yields: mixing and mass loss.

Nucleosynthesis predictions of AGB stellar evolution are confronted with a wide variety of observables. Among these are isotopic ratios of pre-solar grain, now increasingly from multiple measurements of single grains (e.g. Barzyk et al. 2006; Marhas et al. 2007). AGB nucleosynthesis is observable intrinsically in AGB stars (McSaveney et al. 2007, for a recent example), as well in the post-AGB stars, for example in planetary nebulae (Sterling \& Dinerstein 2008) and the H-deficient post-AGB stars (Werner \& Herwig 2006). AGB nucleosynthesis yields are included in galaxy chemical evolution models (e.g. Travaglio et al. 2001; Recchi 2007) and are now helping to address questions such as the "missing satellites" problem of cosmological simulations (Fenner et al. 2006).

$\dagger$ Present address: Dept. of Physics and Astronomy, University of Victoria, Victoria, BC, Canada 
In this paper I will cover work that appeared in the last couple of years, as previous work is reviewed in some detail in the above mentioned and similar references. In Sect. 2 I will discuss the situation of mixing and mass loss in the context of one-dimensional stellar evolution models. Sect. 3 is devoted to simulations in the very low metallicity regime. In both sections I will highlight which problems can now be addressed using 3D hydrodynamic simulations of parts of the star, and this topic will be addressed in Sect. 4, to be followed by some concluding remarks.

\section{Progress in 1D AGB evolution models}

\subsection{Convection}

AGB star evolution simulations and the resulting observable predictions are extremely sensitive to the mixing physics, and in this regard AGB stars are no different than for example massive stars. And as in other types of stars several physical processes contribute to the overall mixing.

Foremost of course is convection with fluid velocities, for example in the He-shell flash convection, of several $\mathrm{km} / \mathrm{s}$. Nuclear physics measurements are now providing the data to analyse s-process branchings that are sensitive to the details of the convective flow in the He-shell flash convection zone (Reifarth et al. 2004; Mohr et al. 2007; Heil et al. 2008), and a more realistic treatment of convective flows than possible through the mixing-length theory is needed to take full advantage of these new measurements.

The role of mixing at convective boundaries has been extensively discussed over the past years, e.g. Mowlavi (1999), or for a more recent summary Herwig (2005). The bottom line is that the important third dredge-up can only be obtained in stellar evolution models with some kind of mixing at the bottom of the convective boundary, and various physical models and numerical algorithms are employed. Also, of course, the ${ }^{13} \mathrm{C}$ pocket as the source of neutrons in the s-process depends on some mixing triggered by convection at the time of the third dredge-up. While it is clear that the source of this convective boundary mixing must be a hydrodynamic instability the exact nature of this mixing is still unclear. But in any case, the effect of this instability must be taken into account during the dredge-up phase of AGB stars of all masses and metallicities (see Sect. 3).

In Fig. 1 I show core-mass evolutions for several AGB tracks with certain choices for the overshoot parameter at the bottom of the pulse-driven convection zone $(=$ He-shell flash convection zone) $f_{\mathrm{PDCZ}}$, and the bottom of the envelope concection during the third dredge-up, $f_{\mathrm{CE}}$. The former parameter is indicated by hydrodynamic He-shell flash convection simualations (Herwig et al. 2007), while the latter reproduces the observed s-process strength of intrinsic AGB stars (Lugaro et al. 2003). Stellar evolution models show that the choices for convective boundary mixing sensitively affect yields, for example through the thermal pulse strength and the dredge-up efficiency.

The properties of envelope convection determines the yields and quantitative evolution as well. This has been pointed out by Boothroyd \& Sackmann (1988) in the context of the mixing-length theory, and by Mazzitelli et al. (1999) when applying the full-spectrum turbulence theory instead to AGB stars. The synthetic AGB models by Marigo et al. (1996) considered the mixing-length parameter uncertainty as well. Nevertheless all other contemporary AGB stellar evolution models are calculated with a mixing-length parameter calibrated to reproduce the parameters of the sun although there is no reason that that mixing-length parameter should be universally applicable to all convection zones in all phases of stellar evolution. Results by Porter \& Woodward (2000) and McSaveney et al. (2007) hint at a possibly larger mixing length parameter compared to the one that best 


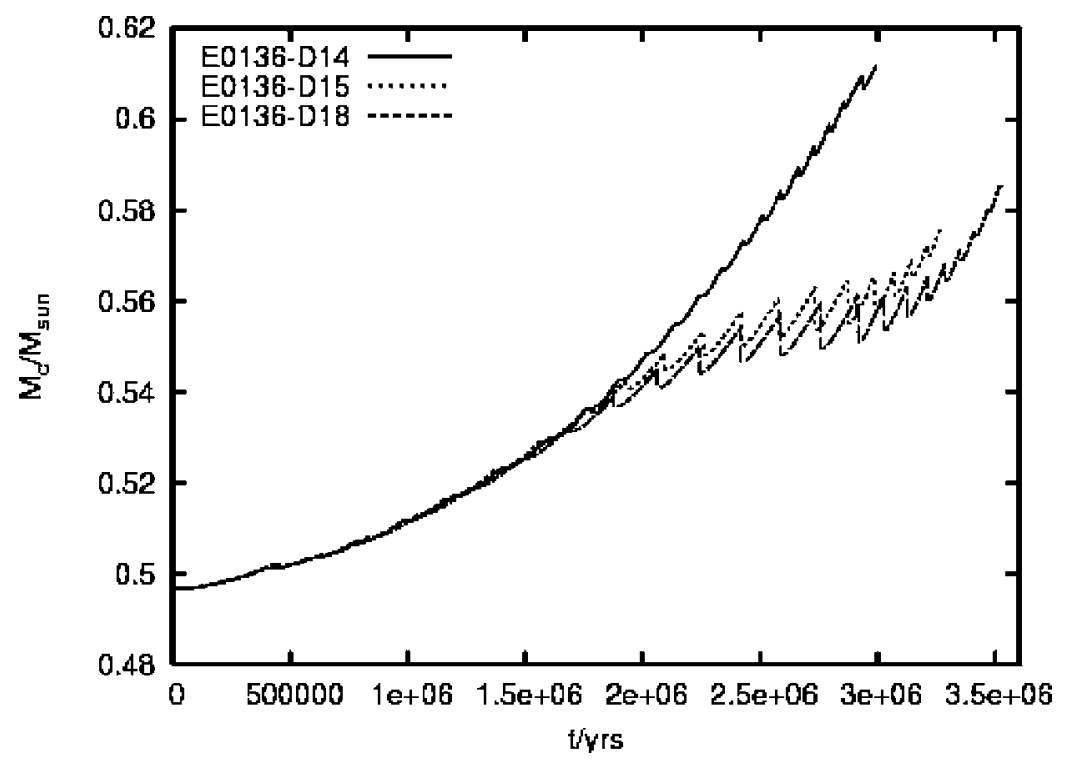

Figure 1. Core mass evolution for initial mass $2 \mathrm{M}_{\odot}$ and $\mathrm{Z}=0.01$ for three choices of convective parameters. D14: $f_{\mathrm{PDCZ}}=0.008, f_{\mathrm{CE}}=0.128$ and $\alpha_{\mathrm{MLT}}=1.7 ; \mathrm{D} 15$ : same as D14 but $\alpha_{\mathrm{MLT}}=2.5 ;$ D18: same as D15 but revised nuclear reaction rate for ${ }^{14} N(p, \gamma)(0.64 \times \mathrm{NACRE}$, Herwig \& Austin 2004).

reproduces the solar parameters. Figure 1 shows the core mass evolution for cases with sun-calibrated mixing-length parameter $\alpha_{\mathrm{MLT}}$ (case D14) and increased envelope convection efficiency. The effect is significant as deeper third dredge-up (as in the D15 and D18 sequence) implies a larger enrichment in processed material. The calibration of $\alpha_{\mathrm{MLT}}$ for AGB stars certainly desereves more attention, for example through well observed giants in the Magelanic Clouds. Additionally, $\alpha_{\mathrm{MLT}}$ needs to be determined from convective envelope simulations like those by Porter \& Woodward (2000), maybe with updated input physics, or more recently by Freytag \& Höfner (2008).

I will discuss the particular difficulties of convective boundaries at very low metalicity in Sect. 3.

\subsection{Other mixing sources}

As reviewed in Herwig (2005) there is numerous observational evidence that AGB stars experience some mixing through the radiative layer between the convective envelope and the H-burning shell (see Uttenthaler et al. 2007, for a recent example). Recently, two physical processes have been investigated in this context.

Eggleton et al. (2006) reported a long overlooked, but absolutely necessary source of mixing, resulting from the reduction of the mean molecular weight through the nuclear reaction ${ }^{3} \mathrm{He}\left({ }^{3} \mathrm{He}, 2 p\right){ }^{4} \mathrm{He}$ which creates locally some boyuancy leading to mixing. The authors make quite correctly the point that the mixing from this $\mu$-effect is robust and has to happen. It is not optional and not based on rather uncertain phsyics assumption. As shown in this conference this $\mu$ mixing can have observable effects in AGB stars as well (this vol., Cantiello \& Langer 2008), and it remains to be seen if there is still a need for additional physical processes as a source of mixing between the H-shell and the bottom of the convection zone, such as magnetic field induced mixing proposed by Busso et al. (2007), in order to accomodate observations. It is interesting to note that the $\mu$-mixing 
may be very efficient at extremely low metallicity as ${ }^{3} \mathrm{He}$ is a primordial $\mathrm{BBN}$ isotope and relatively overabundant at extremely low metallicity.

Rotation has been long suspected to play an important role in AGB stars, but this seems not to be the case, at least according to current simulations of AGB stars including the effect of rotation. Accordingly, rotation is not responsible for the formation of the ${ }^{13} \mathrm{C}$ pocket $\uparrow$, and indeed seems to rather prohibit the s process during the interpulse phase even if a ${ }^{13} \mathrm{C}$ pocket has been assumed to form through some other mixing process (Herwig et al. 2003). Recently, Suijs et al. (2008) reported that the additional inclusion of magnetic fields leads to an enhanced angular momentum transport and eventually reduced AGB core spin rates, bringing simulations to better agreement to asterioseismologically observed white dwarf rotation rates. Whether magnetic fields can also resolve the difference between rotating model s-process predictions and observations quantitatively remains to be seen.

In addition to convection, thermohaline $\mu$-mixing, rotation and magnetic fields, the contribution of internal gravity waves (Press 1981) to angular momentum redistribution and mixing has been investigated. We certainly observe the excitation of these waves in the vicinity of He-shell flash convection in 2D and 3D compressible hydrodynamic simulations (see Sect. 4, Herwig et al. 2006). Again, this type of mixing may be related to the formation of the ${ }^{13} \mathrm{C}$ pocket (Denissenkov \& Tout 2003) or to the global redistribution of angular momentum or chemical species (Talon \& Charbonnel 2008).

\subsection{Mass loss and opacities}

In recent years progress has been also made in the area of mass loss. While it has been pointed out that the dist-driven wind scenario is in trouble for stars with $\mathrm{C} / \mathrm{O}<1$ (Höfner 2007) progress has been made for the C-stars through more realistic treatment of radiation in the wind models (Höfner et al. 2003) and by implementing a more realistic treatment of the pulsations now from full 3D hydrodynamic simulations (Freytag \& Höfner 2008).

As an example, recent applications of the new generation of tools to study mass loss in $\mathrm{C}$ stars suggests that the energy injection of pulsations is of greater importance than metallicity, and that C-stars at low metallicity should therefore have similar mass loss as their more metal-rich counterparts (Mattsson et al. 2008).

In order to couple these new mass loss rates to stellar evolution calculations chemistry dependent molecular opacities need to be included to ensure the right surface parameters, and examples of such calculations include those by Cristallo et al. (2007) with an emphasis on metal-poor AGB stars. It seems that the new mass loss simulations together with a consistent treatment of low-temperature opacities puts a solution of the mass loss problem, as least for C-stars within reach.

\subsection{Nuclear physics}

Nuclear reaction rate uncertainties effect both the yields directly as well as indirectly through the structural evolution. For example, the downward revision of the ${ }^{14} N(p, \gamma){ }^{15} \mathrm{O}$ reaction (e.g. LUNA Collaboration et al. 2006) leads to a notable increase of the third dredge-up efficiency (Herwig \& Austin 2004; Weiss et al. 2005; Herwig et al. 2006). Other work has focused on the yield effect of nuclear reaction rate uncertainties, for example for p-capture rates of the $\mathrm{NeNa}$ and $\mathrm{MgAl}$ chains during $\mathrm{HBB}$ in intermediate-mass AGB stars (Izzard et al. 2007).

$\dagger$ The ${ }^{13} \mathrm{C}$ pocket reported by Langer et al. (1999) is too small in mass to account for observations, as shown by Herwig et al. (2003). 


\section{Evolution at very low metallicity}

The evolution of AGB stars at very low metallicity has received enourmous attention over the past years, mostly due of course to the continuing discovery of more interesting very metal-poor stars (Beers \& Christlieb 2005). As many of the C-enhanced stars of extremely low metallicity (CEMP) are believed to be companions of and polluted by former AGB stars (e.g. Lucatello et al. 2005; Lugaro et al. 2008; Stancliffe et al. 2007, see also Pols, this vol.) we are confronted with the investigation of stellar evolution at very low metal content in stars in general, and in AGB stars especially.

One feature is particularly noteworthy, as it causes serious problems for the traditional one-dimensional, spherically symmetric stellar evolution approach. At very low metal content the entropy barrier between $\mathrm{He}$ - and $\mathrm{H}$-shell burning layers is reduced and mixing of $\mathrm{H}$ into very hot ${ }^{12} \mathrm{C}$-rich He-shell burning ash material may trigger violent flash-like convective-reactive phases. Such events can be found in massive stars (e.g. non-rotating: Heger \& Woosley 2008), and sometimes induced by rotation (Hirschi et al. 2005; Ekström et al. 2008).

In AGB stars two variants of the convective-reactive theme are known. The H-ingestion flash in low-metallicity AGB stars involves entrainment of $\mathrm{H}$ into the He-shell flash convection zone (Fujimoto et al. 2000; Herwig 2003; Iwamoto et al. 2004; Campbell 2007, and many older references there). The hot dredge-up (Herwig 2004; Goriely \& Siess 2004 ) is encountered if even only a small fraction of the convective boundary mixing that was introduced to create a $\mathrm{H}_{-}{ }^{12} \mathrm{C}$ partial mixing zone for the ${ }^{13} \mathrm{C}$ is also included at the bottom of the convective envelope during the dredge-up in slightly more massive $\left(M>2 \ldots 3 \mathrm{M}_{\odot}\right)$ AGB stars of very low metalicity $([\mathrm{Fe} / \mathrm{H}]<-2)$. In both cases the commonly used mixing-length theory to treat convection and derive a diffusion coefficient for mixing is not applicable as it does not account for injection of nuclear energy on a hydrodynamic time scale of the convective flow. Woodward et al. (2008) discuss in some detail this problematic situation, which requires 3-dimensional hydrodynamics simulations to be properly addressed.

\section{3D hydrodynamic simulations of AGB interiors}

Stellar hydrodynamics has overwhelmingly been concerned with the stellar surface layers, atmospheres and shallow envelopes (e.g. Nordlund 1982; Stein \& Nordlund 1998; Porter \& Woodward 2000) because the Mach numbers are larger near the surface, making explicit, compressible simulations feasible.

But convective boundaries in surface convection zones behave radically different compared to the deep stellar interior. For example, the hydrodynamic simulations of surface convection in A-type stars and white dwarfs (Freytag et al. 1996) show that coherent convective systems are accelerated in the unstable zone and then transition into the neighbouring formally stable layer only to reverse direction after crossing a significant fraction of a pressure scale height. The situation is very different in the deep interior of AGB stars. Plane-parallel "box-in-a-star" hydrodynamic simulations of He-shell flash convection in two and three dimenstions (Herwig et al. 2006) show rather stiff convective boundaries. While convective systems occupy the entire vertical span of the unstable zone of approx. $4500 \mathrm{~km}$ in these simulations the convective bounadries are poorly resolved at grid size of $20-50 \mathrm{~km}$. Nevertheless, careful analysis of simulatons with a resolution of $13.5 \mathrm{~km}$ showed that overshooting at the bottom of the convection zone is as expected small (Freytag \& Herwig 2008; Herwig et al. 2007), and to first order in agreement 
with stellar evolution constraints from H-deficient post-AGB stars (Werner \& Herwig 2006).

The upper He-shell flash convection boundary is not important in normal thermal pulses, but in those AGB stars of very low metallicity and on the post-AGB where the $\mathrm{H}$-ingestion flashes take place, the upper boundary is of utmost importance as it is here that the H-entrainment will take place. Woodward et al. (2008) reported preliminary simulations zooming in with a grid of up to $1024^{3}$ on the $100 \mathrm{~km}$ above and below the top convective boundary. These simulations show a significant entrainment of $\mathrm{H}$ into the C-rich intershell. It is the competition of mixing accross the bounadry and largerscale convective transport that will determine the inhomogeneities of the fuel-mix, and eventually determine the properties of the H-burning front. For example, larger horizontal inhomogeneities of mixed-down $\mathrm{H}$ will lead to a more patchy energy generation and a broader H-burning front, which may in the end be permeable for mixing of the resulting ${ }^{13} \mathrm{C}$ to the bottom of the He-shell flash convection zone. Here the lifetime of ${ }^{13} \mathrm{C}$ against $\alpha$ capture, and thereby neutron release, is only seconds. By definition spherically-symmetric simulations can not account for any of these horizontal inhomogeneities, which makes them unsuitable for predictive simulations of the convective-reactive phases of evolution in stars, including the hot dredge-up discussed in Sect. 3.

\section{Conclusions}

In this review I have discussed some aspects of AGB stellar evolution that are currently been worked on. Significant progress has been made in better understanding the effect of the various physical processes that may be responsible for mixing. In some areas we are now (or very soon) getting better agreement with observations, for example in models including rotation and additional angular momentum transport. However, predicted white dwarf rotation rates are still typically higher by an order of magnitude compared to observed rotation rates, even including the effect of magnetic fields. Then, on the underlying physics there is in some areas no consesus yet, as demonstrated recently by the debate over the Tayler-Pitts-Spruit dynamo supposed to provide substantial mixing and angular momentum transport (Zahn et al. 2007). Furthermore, the effect of some processes discussed maybe virtually indistinguishable in observations. Internal gravity waves and magnetic fields may have similar consquences, making it maybe at this time difficult to validate. However, the recent trajectory of progress clearly indicates that at least in the area of convection we should be able to finally resolve issues surrouding the mechanism and efficiency of overshooting in the next couple of years. This, together with progress in mass loss, molecular opacitiy and the thermohaline $\mu$-mixing will already provide for a significantly improved new standard model of AGB stars which should emerge in the near future.

\section{Acknowledgements}

I have to thank Bernd Freytag for his most important contributions to our collaborative work on He-shell flash convection. I am indebted to Paul Woodward, David Porter and their students for an extremely enjoyable, ongoing collaboration. I would like to thanks Marco Pignatari for his suggestions on nucleosynthesis and grains. This research was supported by a Marie Curie International Reintegration Grant withing the 6th European Community Framework Programme, grant MIRG-CT-2006-046520 as well as through the Joint Institute for Nuclear Astrophysics, NSF grant PHY 02-16783. 


\section{References}

Barzyk, J. G., Savina, M. R., Davis, A. M., Gallino, R., Pellin, M. J., Lewis, R. S., Amari, S., \& Clayton, R. N. 2006, New Astronomy Review, 50, 587

Beers, T. C. \& Christlieb, N. 2005, ARAA, 43, 531

Blöcker, T., Herwig, F., \& Driebe, T. 2000, in The changes in abundances in AGB stars, ed. F. D'Antona \& R. Gallino, Mem. Soc. Astron. Ital., in press, astro-ph/0002455

Boothroyd, A. I. \& Sackmann, I.-J. 1988, ApJ, 328, 671

Busso, M., Gallino, R., \& Wasserburg, G. J. 1999, ARA\&A, 37, 239

Busso, M., Wasserburg, G. J., Nollett, K. M., \& Calandra, A. 2007, ApJ, 671, 802

Campbell, S. W. 2007, PhD thesis, Monash University, Australia, http://www.asiaa.sinica.edu. tw/simcam/work/phd/thesis-SimonCampbell-A4singleside-6Jul07.pdf

Cantiello, M. \& Langer, N. 2008, ArXiv e-prints, 806

Cristallo, S., Straniero, O., Lederer, M. T., \& Aringer, B. 2007, ApJ, 667, 489

Denissenkov, P. A. \& Tout, C. A. 2003, MNRAS, 340, 722

Eggleton, P. P., Dearborn, D. S. P., \& Lattanzio, J. C. 2006, Science, 314, 1580

Ekström, S., Meynet, G., Chiappini, C., Hirschi, R., \& Maeder, A. 2008, ArXiv e-prints, 807

Fenner, Y., Gibson, B. K., Gallino, R., \& Lugaro, M. 2006, ApJ, 646, 184

Forestini, M. \& Charbonnel, C. 1997, A\&AS, 123, 241

Freytag, B. \& Herwig, F. 2008, ApJ, in prep

Freytag, B. \& Höfner, S. 2008, A\&A, 483, 571

Freytag, B., Ludwig, H.-G., \& Steffen, M. 1996, A\&A, 313, 497

Fujimoto, M. Y., Ikeda, Y., \& Iben, I., J. 2000, ApJ Lett., 529, L25

Goriely, S. \& Siess, L. 2004, A\&A, 421, L25

Heger, A. \& Woosley, S. E. 2008, ArXiv e-prints, 803

Heil, M., Winckler, N., Dababneh, S., Käppeler, F., Wisshak, K., Bisterzo, S., Gallino, R., Davis, A. M., \& Rauscher, T. 2008, ApJ, 673, 434

Herwig, F. 2003, in CNO in the Universe, ASP Conf. Ser. astro-ph/0212366

Herwig, F. 2004, ApJ, 605, 425

-. 2005, ARAA, 43

Herwig, F. \& Austin, S. M. 2004, ApJ Lett., 613, L73

Herwig, F., Austin, S. M., \& Lattanzio, J. C. 2006, Phys. Rev. C., 73, 025802

Herwig, F., Freytag, B., Fuchs, T., Hansen, J. P., Hueckstaedt, R. M., Porter, D. H., Timmes, F. X., \& Woodward, P. R. 2007, in Astronomical Society of the Pacific Conference Series, Vol. 378, Why Galaxies Care About AGB Stars: Their Importance as Actors and Probes, ed. F. Kerschbaum, C. Charbonnel, \& R. F. Wing, 43

Herwig, F., Freytag, B., Hueckstaedt, R. M., \& Timmes, F. X. 2006, ApJ, 642, 1057

Herwig, F., Langer, N., \& Lugaro, M. 2003, ApJ, 593, 1056

Hirschi, R., Meynet, G., \& Maeder, A. 2005, A\&A, 443, 581

Höfner, S. 2007, in Astronomical Society of the Pacific Conference Series, Vol. 378, Why Galaxies Care About AGB Stars: Their Importance as Actors and Probes, ed. F. Kerschbaum, C. Charbonnel, \& R. F. Wing, 145-+

Höfner, S., Gautschy-Loidl, R., Aringer, B., \& Jørgensen, U. G. 2003, A\&A, 399, 589

Iben, Jr., I. \& Renzini, A. 1983, ARA\&A, 21, 271

Iwamoto, N., Kajino, T., Mathews, G. J., Fujimoto, M. Y., \& Aoki, W. 2004, ApJ, 602, 378

Izzard, R. G., Lugaro, M., Karakas, A. I., Iliadis, C., \& van Raai, M. 2007, A\&A, 466, 641

Karakas, A. \& Lattanzio, J. C. 2007, Publications of the Astronomical Society of Australia, 24, 103

Langer, N., Heger, A., Wellstein, S., \& Herwig, F. 1999, A\&A, 346, L37

Lattanzio, J. 1989, in Evolution of Peculiar Red Giants, ed. H. R. Johnson \& B. Zuckermann, IAU Symp. 106 (Cambridge University Press), 161

Lattanzio, J. C. \& Boothroyd, A. I. 1997, in Astrophysical Implications of the Laboratory Study of Presolar Materials, ed. T. Bernatowitz \& E. Zinner (AIP Conf. Ser.), 85

Lucatello, S., Tsangarides, S., Beers, T. C., Carretta, E., Gratton, R. G., \& Ryan, S. G. 2005, ApJ, 625, 825 
Lugaro, M., de Mink, S. E., Izzard, R. G., Campbell, S. W., Karakas, A. I., Cristallo, S., Pols, O. R., Lattanzio, J. C., Straniero, O., Gallino, R., \& Beers, T. C. 2008, A\&A, 484, L27

Lugaro, M., Herwig, F., Lattanzio, J. C., Gallino, R., \& Straniero, O. 2003, ApJ, 586, 1305

LUNA Collaboration, Lemut, A., Bemmerer, D., Confortola, F., Bonetti, R., Broggini, C., Corvisiero, P., Costantini, H., Cruz, J., Formicola, A., Fülöp, Z., Gervino, G., Guglielmetti, A., Gustavino, C., Gyürky, G., Imbriani, G., Jesus, A. P., Junker, M., Limata, B., Menegazzo, R., Prati, P., Roca, V., Rogalla, D., Rolfs, C., Romano, M., Rossi Alvarez, C., Schümann, F., Somorjai, E., Straniero, O., Strieder, F., Terrasi, F., \& Trautvetter, H. P. 2006, Physics Letters B, 634, 483

Marhas, K., Hoppe, P., \& Ott, U. 2007, "Meteoritics \& Planetary Science", 42, 1043

Marigo, P., Bressan, A., \& Chiosi, C. 1996, A\&A, 313, 545

Mattsson, L., Wahlin, R., Höfner, S., \& Eriksson, K. 2008, A\&A, 484, L5

Mazzitelli, I., D'Antona, F., \& Ventura, P. 1999, A\&A, 348, 846

McSaveney, J. A., Wood, P. R., Scholz, M., Lattanzio, J. C., \& Hinkle, K. H. 2007, MNRAS, 378,1089

Mohr, P., Käppeler, F., \& Gallino, R. 2007, Phys. Rev. C., 75, 012802

Mowlavi, N. 1999, A\&A, 344, 617

Nordlund, A. 1982, A\&A, 107, 1

Porter, D. H. \& Woodward, P. R. 2000, ApJS, 127, 159

Press, W. H. 1981, ApJ, 245, 286

Recchi, S. 2007, in Astronomical Society of the Pacific Conference Series, Vol. 378, Why Galaxies Care About AGB Stars: Their Importance as Actors and Probes, ed. F. Kerschbaum, C. Charbonnel, \& R. F. Wing, 364-+

Reifarth, R., Käppeler, F., Voss, F., Wisshak, K., Gallino, R., Pignatari, M., \& Straniero, O. 2004, ApJ, 614, 363

Stancliffe, R. J., Glebbeek, E., Izzard, R. G., \& Pols, O. R. 2007, A\&A, 464, L57

Stein, R. F. \& Nordlund, A. 1998, ApJ, 499, 914

Sterling, N. C. \& Dinerstein, H. L. 2008, ApJS, 174, 158

Suijs, M. P. L., Langer, N., Poelarends, A.-J., Yoon, S.-C., Heger, A., \& Herwig, F. 2008, A\&A, 481, L87

Talon, S. \& Charbonnel, C. 2008, A\&A, 482, 597

Travaglio, C., Gallino, R., Busso, M., \& Gratton, R. 2001, ApJ, 549, 346

Uttenthaler, S., Lebzelter, T., Palmerini, S., Busso, M., Aringer, B., \& Lederer, M. T. 2007, A\&A, 471, L41

Weiss, A., Serenelli, A., Kitsikis, A., Schlattl, H., \& Christensen-Dalsgaard, J. 2005, ArXiv Astrophysics e-prints

Werner, K. \& Herwig, F. 2006, PASP, 118, 183

Woodward, P., Herwig, F., Porter, D., Fuchs, T., Nowatzki, A., \& Pignatari, M. 2008, in American Institute of Physics Conference Series, Vol. 990, First Stars III, 300-308

Zahn, J.-P., Brun, A. S., \& Mathis, S. 2007, A\&A, 474, 145

\section{Discussion}

\section{F. KUPKA:}

(1) Considering that the physical conditions for which the f-parameter model was derived for and the He-shell flash scenario, with all these new and different processes going on in that case - why do you still use the f-parameter model, since its physical basis for that scenario is certainly even less well founded than MLT? It cannot be more than just a mathematical fit formula.

(2) As you have shown with one of your movies, if one does global simulations for this problem, on has to account for large and complex inhomogeneities in the physical conditions at a given radius. It would help to have a table to compare the scales of the box-in-a-star simulations to the global ones. To tie the two types of simulations together to eventually derive predictive models is quite some work - which you may have already studied? 
F. HERWIG:

(1) Freytag et al. (1996) showed, based on their simulations, that (i) the overshooting fluid flows lead to diffusive spreading of hot particle ensembles and (ii) that the corresponding diffusion coefficients decrease exponentially with the distance from the convective boundary. They determined the exponential delay parameter "f" for A type and white dwarf convection from their simulations as $\mathrm{f}=0.25$ and 1.0 , respectively. Already from their paper you can see that although both conventional zones show a similar qualitative exponential overshoot behavior, which are quantitatively represented by different $f$. It is therefore not at all surprising that once we put this mathematical expression for the overshoot behavior into a stellar evolution code we have get again different f-values at different convective boundaries. In some cases we can not even be certain that the physics of convective boundary mixing is the same as in Freytag's simulation. In the deep interior we might rather encounter turbulent entrainment (see Casey Meakin's talk). In that case the f-parameter model still gives you a convenient tool to estimate the effect of convective boundary mixing in 1D-stellar evolution. As a time and depth dependent overshooting implementation it allows us for example to study convective boundary mixing for the formation of the ${ }^{13} \mathrm{C}$-pocket on the hot dredge-up situation in massive, low-Z AGB stars. So, I think it is quite a useful concept. So why do I still use the simple f-parameter model for He-shell flash convection? The first step to either determine $\mathrm{f}$ or improve the mixing model was to start doing the He-shell flash convection simulations. I think we still have to do more work on those. But Bernd Freytag has done a preliminary determination of f-values at the top and bottom of the convective shell, and I showed the results. Interestingly, the f-value we found for the bottom of the He-shell flash convection zone does not obviously violate observables. But we have to check this more carefully.

(2) Yes, I should have explained the geometric setup of the box-in-a-star simulations. The information is fully given in Herwig et al. (2006). 